\title{
Necessary and Sufficient Conditions on State Transformations That Preserve the Causal Structure of LTI Dynamical Networks
}

Chi Ho Leung

Brigham Young University

Follow this and additional works at: https://scholarsarchive.byu.edu/etd

Part of the Physical Sciences and Mathematics Commons

\section{BYU ScholarsArchive Citation}

Leung, Chi Ho, "Necessary and Sufficient Conditions on State Transformations That Preserve the Causal Structure of LTI Dynamical Networks" (2019). Theses and Dissertations. 7413.

https://scholarsarchive.byu.edu/etd/7413

This Thesis is brought to you for free and open access by BYU ScholarsArchive. It has been accepted for inclusion in Theses and Dissertations by an authorized administrator of BYU ScholarsArchive. For more information, please contact ellen_amatangelo@byu.edu. 
Necessary and Sufficient Conditions on State Transformations That Preserve the Causal Structure of LTI Dynamical Networks

Chi Ho Leung

A thesis submitted to the faculty of Brigham Young University

in partial fulfillment of the requirements for the degree of

Master of Science

Sean Warnick, Chair

David Wingate

Mark Transtrum

Jonathan Sillito

Seth Holladay

Department of Computer Science

Brigham Young University

Copyright (C) 2019 Chi Ho Leung

All Rights Reserved 


\begin{abstract}
Necessary and Sufficient Conditions on State Transformations That Preserve the Causal Structure of LTI Dynamical Networks

Chi Ho Leung

Department of Computer Science, BYU

Master of Science

Linear time-invariant (LTI) dynamic networks are described by their dynamical structure function, and generally they have many possible state space realizations. This work characterizes the necessary and sufficient conditions on a state transformation that preserves the dynamical structure function, thereby generating the entire set of realizations of a given
\end{abstract} order for a specific dynamic network.

Keywords: linear systems, state-space methods, necessary conditions, sufficient conditions, state transformation, causal structure, linear time-invariant dynamic networks, dynamical structure function, specific dynamic network, state space realizations, computational modeling, mathematical model, aerospace electronics, transfer functions, structural engineering, heuristic algorithms 


\section{ACKNOWLEDGMENTS}

Thanks Dr. Warnick for his support and helping me throughout the process. 


\section{Table of Contents}

List of Figures $\quad$ vi

1 Introduction $\quad 1$

1.1 Dynamic Systems . . . . . . . . . . . . . . . . . . . 1

1.1.1 State Space Mechanistic Models . . . . . . . . . . . . . . . . . 1

1.1.2 Black Box Transfer Function . . . . . . . . . . . . . . . . 2

1.1.3 Dynamical Structure Functions . . . . . . . . . . . . . . . 3

1.2 Problem Formulation and Contribution . . . . . . . . . . . . . . 7

1.3 Why Do We Care? . . . . . . . . . . . . . . . . . . . 7

1.3.1 Equivalent Classes of Systems . . . . . . . . . . . . . . . 7

1.3.2 Representations of System Structures . . . . . . . . . . . . . . . . 9

1.3.3 Dynamical Structure Function, Its Applications and Theoretical Developments ........................... 10

1.3.4 Designing Stabilizing Distributed Controllers . . . . . . . . . . . . 11

2 Background $\quad 12$

2.1 Causality . . . . . . . . . . . . . . . . . . . . 12

2.1.1 Causality as Variable Dependency . . . . . . . . . . . . . . . . . . 12

2.1.2 Causality as Arrow of Time . . . . . . . . . . . . . . 13

2.1.3 Dynamical Structure Function as a Representation of Causal Dependency Graph Between Signals . . . . . . . . . . . . . 15

2.2 Semantics . . . . . . . . . . . . . . . . . . . . 15 
2.3 Problem Decomposition and Reduction . . . . . . . . . . . . . . 16

2.3.1 Sufficient Conditions on State Transformations, $T$, that Preserve $(W, V) 16$

2.3.2 Extended Definition of Dynamical Structure Function and Reduction

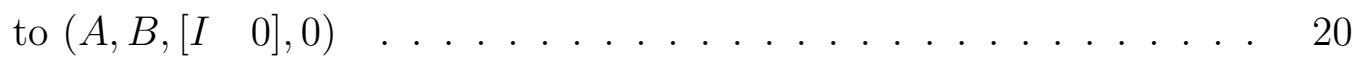

2.3.3 Similarity Transform of the Set of Reduced State Space Models . . . 22

3 Main Result $\quad 24$

3.1 Structural Parameters of a System _ . . . . . . . . . . . . . . . 24

3.2 The Necessary and Sufficient Condition on Transformation $T$ that Preserves

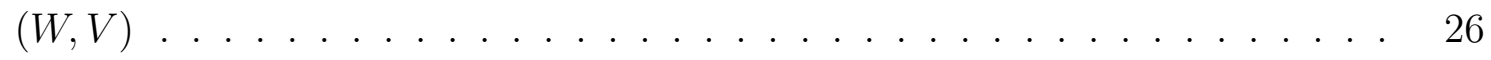

3.3 Equivalent classes of $(W, V)$ characterized by the same $(Q, P) \ldots 31$

4 Conclusion 33

$\begin{array}{lr}\text { References } & 34\end{array}$ 


\section{List of Figures}

1.1 A state space model with 3 inputs, 5 states and 2 outputs. . . . . . . . . 2

1.2 A transfer function with 3 inputs and 2 outputs. . . . . . . . . . . . 3

1.3 A dynamical structure function with 3 inputs and 2 outputs. . . . . . . . . 4

2.1 An example of compressed dependency graph of signals and expanded dependency graph of variables. . . . . . . . . . . . . . . . . . 14

2.2 Graphical illustration of reducing $(A, B, C, D)$ to $\left(\hat{A}, \hat{B},\left[\begin{array}{ll}I & 0\end{array}\right], \hat{D}\right)$ when defin-

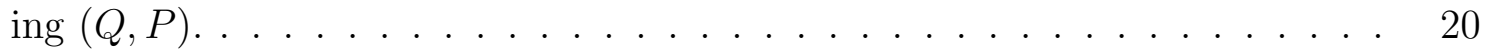




\section{Chapter 1}

\section{Introduction}

Dynamic systems have been studied in various disciplines of science. In recent years, this class of models also gains the attention of the machine learning community [3, 19]. This work studies an important class of computation models, which is known as linear time invariant(LTI) dynamical systems, and characterizes these models by partitioning them into structurally equivalent classes.

\subsection{Dynamic Systems}

There exist various computation models with various level of abstraction; we will introduce some of them here. We will focus our discussion on continuous linear time invariant dynamic system from now on.

\subsubsection{State Space Mechanistic Models}

A state space model of continuous linear time invariant model illustrated in fig(1.1) can be formulated as a system of differential equations:

$$
\begin{aligned}
& \dot{x}(t)=A x(t)+B u(t) \\
& y(t)=C x(t)+D u(t),
\end{aligned}
$$

where $\dot{x}(t) \in \mathbb{R}^{n}$ is the first order derivative of state variable $x(t) \in \mathbb{R}^{n}, u(t) \in \mathbb{R}^{l}$ is the input to the system and $y(t) \in \mathbb{R}^{m}$ is the output of the system at time $t$. The system is said to be full state feedback if $\operatorname{rank}(C)=n$, meaning all information of the state variable $x$ 


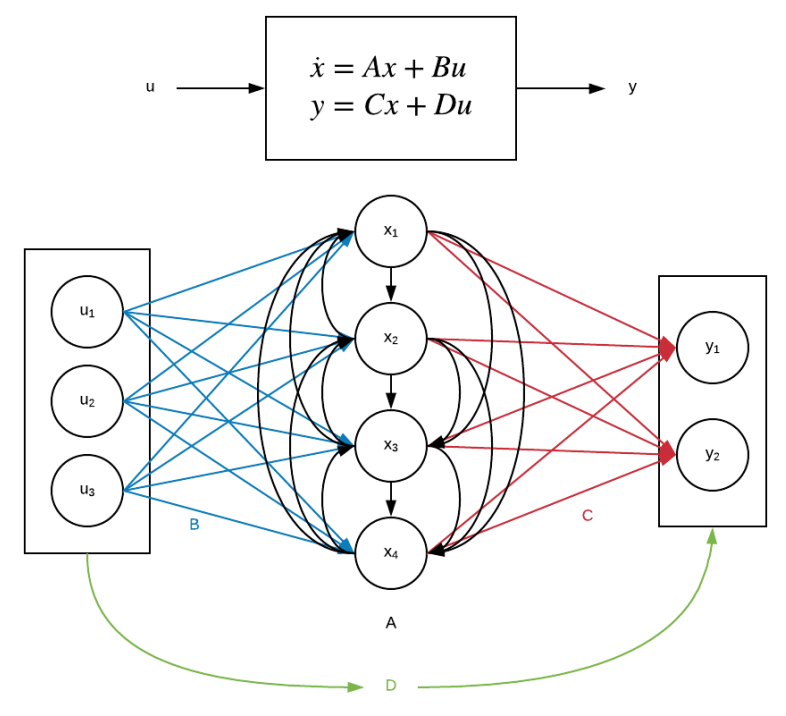

Figure 1.1: A state space model with 3 inputs, 5 states and 2 outputs.

is captured in the output. Furthermore, most physical systems that arise in nature and in engineering have the matrix $D=0$ because information often takes time to transmit from input to output within a system. A system with non-zero $D$ may be associated with some ill-posed properties, such as circular reasoning about the system's initial condition [1].

\subsubsection{Black Box Transfer Function}

A transfer function, as illustrated in fig(1.2)is an abstraction of a set of state space models $(A, B, C, D)$. It abstracts away the interconnections between state variables $x$ and casts the model into a simple mapping between input and output signals. It can be obtained through derivation from a state space model $(A, B, C, D)$ as:

$$
Y(s)=\hat{G}(s) U(s), \quad \hat{G}(s)=C(s I-A)^{-1} B+D,
$$




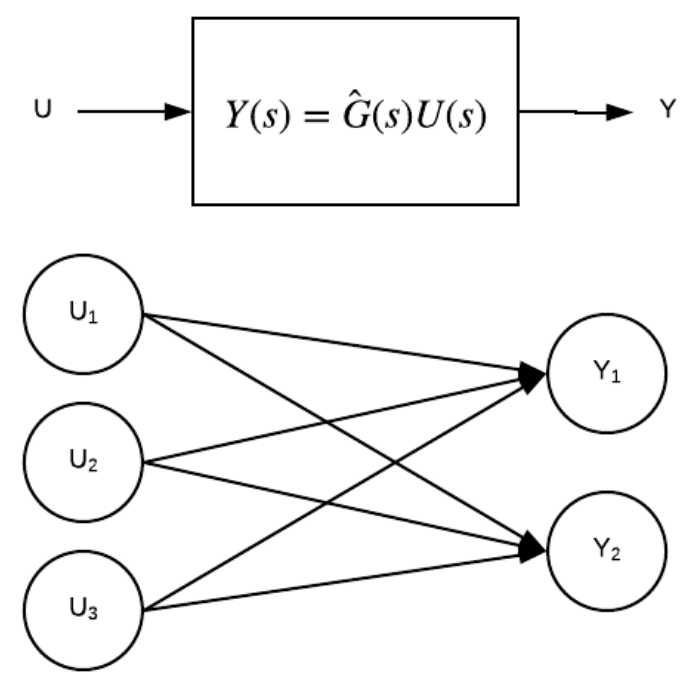

Figure 1.2: A transfer function with 3 inputs and 2 outputs.

where $s \in \mathbb{C}$ is a Laplace variable used in transforming the state space model from the domain of time to the domain of frequency:

$$
\mathcal{L}[f(t)]=\int_{0}^{\infty} e^{-s t} f(t) d t=F(s)
$$

where $U(s) \in \mathbb{C}^{l}, Y(s) \in \mathbb{C}^{m}$ represents the input and output signal with a certain frequency and exponential envelope. Notice that each entry in $\hat{G}(s)$ is a rational polynomial in $s$, and multiplication of $\hat{G}(s) \cdot U(s)$ in the Laplace domain is equivalent to convolution $F(t) \star G(t)$ in the time domain.

\subsubsection{Dynamical Structure Functions}

There also exists a representation with an abstraction level in between transfer functions and state space models. We call it the dynamical structure function, as illustrated in fig(1.3). It is an abstracted representation of a set of state space models. The dynamical structure 


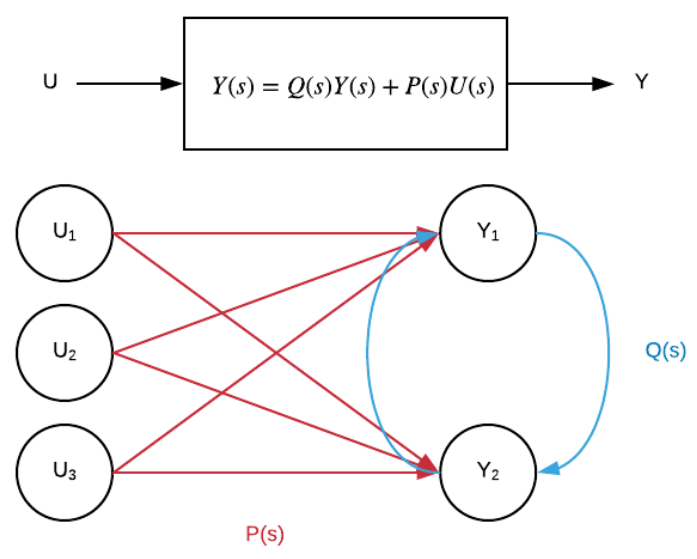

Figure 1.3: A dynamical structure function with 3 inputs and 2 outputs.

function(DSF) can be derived from a state space model, $(A, B, C, D)$ :

$$
\begin{aligned}
& \dot{x}=A x+B u \\
& y=C x+D u .
\end{aligned}
$$

The dynamical structure function of a linear time invariant system is characterized by the following equation [5],

$$
Y(s)=Q(s) Y(s)+P(s) U(s)
$$

and the derivation of $(Q, P)$ from $(A, B, C, D)$ is as follow:

We first partition $(A, B, C, D)$ into block matrices commensurate with the partitioning of manifest variables $Y$ and hidden variables $X$. We assume that $C=\left[\begin{array}{ll}I & 0\end{array}\right]$ and $D=0$, the justification of such assumption has been given in [5] and will be restated again in a later section. By taking Unilateral Laplace transform on (1.1) and introducing the Laplace 
variable $s$, we have:

$$
\begin{aligned}
s\left[\begin{array}{l}
Y \\
X
\end{array}\right] & =\left[\begin{array}{ll}
A_{11} & A_{12} \\
A_{21} & A_{22}
\end{array}\right]\left[\begin{array}{l}
Y \\
X
\end{array}\right]+\left[\begin{array}{l}
B_{1} \\
B_{2}
\end{array}\right] U \\
Y & =\left[\begin{array}{ll}
I & 0
\end{array}\right]\left[\begin{array}{l}
Y \\
X
\end{array}\right] .
\end{aligned}
$$

By expanding equation (1.3), we obtain

$$
\begin{aligned}
& s Y=A_{11} Y+A_{12} X+B_{1} U \\
& s X=A_{21} Y+A_{22} X+B_{2} U
\end{aligned}
$$

Now, we rearrange equation (1.5) to be

$$
X=\left(s I-A_{22}\right)^{-1}\left(A_{21} Y+B_{2} U\right)
$$

and by substituting equation (1.6) into equation (1.4), we have

$$
\begin{gathered}
s Y=A_{11} Y+A_{12}\left(s I-A_{22}\right)^{-1}\left(A_{21} Y+B_{2} U\right)+B_{1} U \\
s Y=\left(A_{11}+A_{12}\left(s I-A_{22}\right)^{-1} A_{21}\right) Y \\
\quad+\left(A_{12}\left(s I-A_{22}\right)^{-1} B_{2}+B_{1}\right) U .
\end{gathered}
$$

Definition 1.1.1 (Dynamical Network Function) Now, we can define $W(s)$ and $V(s)$ as the following,

$$
\begin{gathered}
W(s)=A_{12}\left(s I-A_{22}\right)^{-1} A_{21}+A_{11}, \\
V(s)=A_{12}\left(s I-A_{22}\right)^{-1} B_{2}+B_{1},
\end{gathered}
$$


where $s Y(s)=W(s) Y(s)+V(s) U(s)$. The tuple $(W, V)$ is called the dynamical network function (DNF) of the system.

In this work, we call $(W, V)$ the dynamical network function of the system because it contains all causal linkages of a system including its self-influencing edges. Now, equation (1.8) can be rewritten as

$$
s Y=W(s) Y+V(s) U
$$

For computational convenience, we further manipulate $W(s)$ and $V(s)$ by subtracting the diagonal of $W(s)$ on both side and move $s$ to the right,

$$
(s I-\operatorname{diag}(W(s))) Y=(W(s)-\operatorname{diag}(W(s))) Y+V(s) U
$$

then by moving $(s I-\operatorname{diag}(W(s)))$ to the right, we get

$$
Y=(s I-\operatorname{diag}(W(s)))^{-1}(W(s)-\operatorname{diag}(W(s))) Y+(s I-\operatorname{diag}(W(s)))^{-1} V(s) U
$$

Definition 1.1.2 (Dynamical Structure Function) With equation (1.13), we define $(Q, P)$ as

$$
\begin{aligned}
& Q(s)=(s I-\operatorname{diag}(W(s)))^{-1}(W(s)-\operatorname{diag}(W(s))), \\
& P(s)=(s I-\operatorname{diag}(W(s)))^{-1} V(s) .
\end{aligned}
$$

The tuple $(Q, P)$ is called the dynamical structure function (DSF) of the system.

In contrast with the dynamical network function $(W, V)$, the dynamical structure function $(Q, P)$ contains only the inter-variable structure but not intra-variable structure, since all self-influencing edges are dissolved into other parts of the system. This decision of design grants network reconstruction nice properties, such as reduced number of edges for learning [6]. 


\subsection{Problem Formulation and Contribution}

With the definition of dynamical structure function given above, we can now formulate our problem statement as follow:

Given a system:

$$
\begin{aligned}
& \dot{x}=A x+B u \\
& y=C x+D u,
\end{aligned}
$$

with a dynamical structure function $(Q, P)$, what is the necessary and sufficient condition on a state transformation $T$ such that the new system:

$$
\begin{aligned}
& \dot{x}=T A T^{-1} x+T B u, \\
& y=C T^{-1} x+D u
\end{aligned}
$$

has the same dynamical structure function $(Q, P)$ as the original system. The contribution of this work is the discovery of the necessary and sufficient condition which characterizes state space models realizing the same dynamical structure function.

\subsection{Why Do We Care?}

We will discuss the theoretical importance and some application implications of the problem we have solved in this section. Important related works will also be mentioned in this section.

\subsubsection{Equivalent Classes of Systems}

Changing of basis is one of the fundamental concepts employed by linear algebra, in essence, every matrix composition is some change of basis specified by the column space of the left matrix. 
Definition 1.3.1 (Similar Matrices) Two matrices $A$ and $B$ are similar if there exists an invertible matrix $S$ such that $A=S^{-1} B S$.

Spanning from this definition of similarity is the famous theorem of Jordan Canonical Forms. This theorem proves the existence of an unique similar block diagonal matrix of any given matrix under an algebraically complete field, assumed a certain ordering of its Jordan blocks. It also follows that two matrices are similar if and only if they have the same Jordan Canonical Form. This notion of similarities with respect to some changes of basis has been used along side with other notion of similarity, such as row echelon form with respect to row reduction of matrices. Each of these notions of similarity builds equivalent classes of linear transformations with particular properties in common. In particular, matrices with the same Jordan Canonical Form share the same spectral properties, which means sharing the same set of eigenvalues with corresponding multiplicities.

Kalman in one of his early work [15] lay down some fundamental building blocks for modern control theory, suggested a notion of similar dynamical systems as follow:

Definition 1.3.2 (Algebraically Equivalent Systems) Two continuous-time systems

$$
\left\{\begin{array} { l } 
{ \dot { x } = A x + B u } \\
{ y = C x + D u }
\end{array} \quad \text { or } \quad \left\{\begin{array}{l}
\dot{\bar{x}}=\bar{A} \bar{x}+\bar{B} u \\
y=\bar{C} \bar{x}+\bar{D} u
\end{array}\right.\right.
$$

respectively, are algebraically equivalent if there exists a nonsingular matrix $T$ such that

$$
\bar{A}:=T A T^{-1}, \quad \bar{B}:=T B, \quad \bar{C}:=C T^{-1}, \quad \bar{D}:=D
$$

holds. The corresponding map $\bar{x}=T x$ is called a similarity transformation or an equivalent transformation.

It turns out that algebraically equivalent state space representations are also zero-state equivalent, which means the two matrices exhibit the same transfer function, if they are 
minimal [14], which would imply that the two systems also share the same stability, sensitivity and robustness properties which were spanned from the spectral properties of the $(A, B, C, D)$ matrices.

Because of the set of properties shared within each algebraically equivalent class, and our objective to investigate finer properties like system performances under some coarser requirements such as system stability and robustness, it is natural to build our new characterization on the same set of tools.

\subsubsection{Representations of System Structures}

There are a number of other models $[2,9,17,18,22]$ attempting to capture a system's dynamical structure besides dynamical structure function, some of them are proper subsets of the DSF, for example, linear dynamical graph suggested by Materassi and Innocenti. In their works, $P$ in $(Q, P)$ is replaced by $I$ and assume all input to be i.i.d. random noises. On the other hand, the problem of finding hidden structure of systems have been approached from a statistical point of view, from which a set of techniques related to Bayesian Graphical Models [21] were developed. Although this kind of graphical models are static in nature and do not capture feedback effects of a dynamical system, we have reasons to believe that they are related to dynamical structure functions and some of its closer relatives in the sense that they all attempt to model structures of variables manifested by some phenomena or systems. The differences between the two kind of representations rise because one based its tradition on differential equations while the other found its root in probability theory.

System structures can also be described in terms of its subsystem nature with the modeling technique called linear fractional transformation. In comparison, modeling technique we called dynamical structure function [26] is one that concerns with the signal structure of a system. The main difference between subsystem structures and signal structures is that subsystem structures require a partitioning of systems' state variables into distinct interconnected subsystems, while signal structure allows a hidden state variable to be dissolved 
into multiple subparts of its structural representation. The signal structure representation is more beneficial for learning tasks like network reconstruction, which objective is to identify a system's structure by looking at its manifested behaviors. It is because the cost of learning the signal structure is lower than that of learning the subsystem structure representation, which involves figuring out all the correct partition of state variables. Besides, the sparsity patent of a transfer function can also provide some weak notions of structure, but often time a more informative representation like the dynamical structure function will be more desirable provided with sufficient a priori knowledge about our target system.

Despite of the existence of different structural representations of interconnected systems, dynamical structure functions are chosen as the primary representations of structure in this work because of its appropriate generalizability, complexity and informativity with respect to the set of applications and theoretical works that are in line with our interest.

\subsubsection{Dynamical Structure Function, Its Applications and Theoretical Develop- ments}

One of the primary application and motivation of the development of dynamical structure function is to reconstruct the dynamical influences between manifest variables of a system. This problem has been formulated in [11], and it has been applied to biochemical reaction networks, financial networks [25], and social networks [7].

On the theoretic side, there have been works on characterizing the semantics of dynamical networks, that is the well definedness of dynamical structure function, through investigating their set of state space realizations [5]. Other works like Chetty and Warnick have classified system representations by their hierarchy of computation granularity, from the most abstract manifest structures to the most detailed complete computational structures. Furthermore, there have been works on comparing the information cost of reconstructing state space representations and structured linear fractional transformations with dynamical structure function, and have shown that the cost, which is the amount of a priori information 
needed. It is proven that cost of reconstructing a dynamical structure function is less than that of state space representation and linear fractional transformation. [20] [4]

\subsubsection{Designing Stabilizing Distributed Controllers}

Rai and Warnick has explored the possibility of designing stabilizing distributed controllers in [23], and their results guarantee that either a stabilizing controller can be found, or no stabilizing controllers exist under given configurations. However, a distributed controller discovered by their algorithm is not guarantee to have good performance. In order to search for a controller with high performance, we would need to characterize the set of all state space realizations with the same dynamical structure function given a certain realization. 


\section{Chapter 2}

\section{Background}

This chapter is an in-depth discussion on the significance of discovering characterization of structurally equivalent class of state space models.

\subsection{Causality}

Although there are still doubts in the existence of causality within scientific societies [24], the formal notion of causality has been investigated in various fields $[10,16]$. Pearl has explicitly defended and defined causality in terms of Bayesian networks in [21], and we will borrow his insights on this subject.

\subsubsection{Causality as Variable Dependency}

Consider a simple example, where we are interested in the relationship between the slipperiness of pavement and the state of a sprinkler. We built a predictive model for the problem, and ask three of questions:

1. Prediction (would the pavement be slippery if we find the sprinkler off?)

2. Intervention (would the pavement be slippery if we make sure that the sprinkler is off?)

3. Counterfactuals (would the pavement be slippery had the sprinkler been off, given that pavement is in fact not slippery and the sprinkler is on?)

The ability for our model to answer these questions will depend on the level of causal information encoded in it. A model with only correlation information can only deduce the 
answer for the first type of question, and we can only answer the second type of question adequately if we know the cause and effect relationship between slippery pavement and sprinkler. Pearl suggested that it is by controlled experiments that we are able to discover the causal content of a system to answer the second question. If controlled experiments are not a practical option, then a rich enough dataset will be needed to provide equivalent information as a controlled experiments for discovering all conditional branches and modes of the system with sufficient statistical significance.

The third type of question attacks the untestable blind spot in a dataset. A better example for illustrating the nature and difficulty of this type of question is the clinical trial example. Suppose Joe has participated in a clinical trial of experimental medication and died. A counterfactual question would be: "would Joe be alive had he not been taken the medication given that he has in fact taken the medication and died?" The fact that we could not go back in time and perform the control test for Joe make this question hypothetical and unanswerable. Some "closest world assumptions" need to be made to cast this question into a testable one. For example, let say Joe has a twin brother and he was in the control group of medication at the same time as Joe was taking the medication. Then the result of Joe's twin brother can be used as a closest world approximation to Joe's medication reaction[21]. However, a priori knowledge is needed to induce a proper closest world casting, which requires a well justified scheme for involving external knowledge in predictive models with various complexity and domain specificity.

\subsubsection{Causality as Arrow of Time}

In dynamic system, an operator is said to be causal if the output does not depend on future values of the input. This property can be formalized by introducing a truncation operator $P_{T}:$

$$
P_{T}(u(t))= \begin{cases}u(t) & \text { for } t \leq T \\ 0 & \text { for } t>T\end{cases}
$$


A system with $S(u(t))$ is causal if

$$
P_{T}\left(S\left(P_{T}(u)\right)\right)=P_{T}(S(u)),
$$

that is, the future input of $u$ does not affect the past output of $S$. The reason for introducing this notion of causality is because a signal in a dynamic system can self-influence through time, causality as arrow of time help us distinguish between causal (past does not depend on future), anti-causal (future does not depend on past) and non-causal (future and past are interdependent) systems. It is a way of specifying the structure of the dependency graph between variables across time, hence answering the questions:

1. Is the dependency graph across time directed and acyclic or not?

2. If it is a DAG, is the direction backward in time or forward in time?

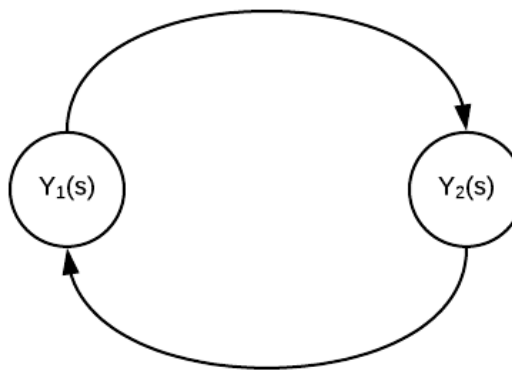

Compressed Representation

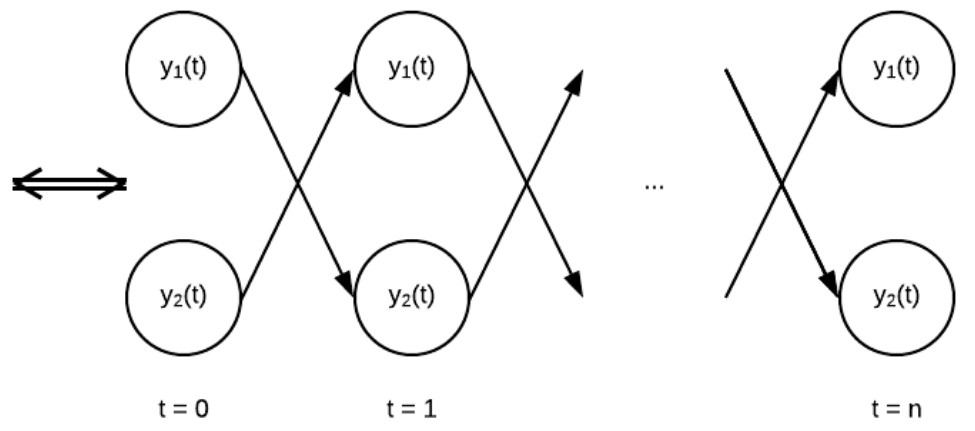

Expanded Representation

Figure 2.1: An example of compressed dependency graph of signals and expanded dependency graph of variables.

Hence a dependency graph with signal as node with seemingly circular reasoning can be unwraps and expands into a DAG as illustrated in fig(2.1). 


\subsubsection{Dynamical Structure Function as a Representation of Causal Dependency Graph Between Signals}

Dynamical structure functions are a pair of complex rational polynomial matrices $(Q(s), P(s))$. Each entry $Q_{i j}$ encodes a mapping from node $j$ to node $i$. Furthermore, it is guaranteed to be strictly causal in the sense of the arrow of time by guaranteeing each rational polynomial has a numerator with lower order than the denominator. Thus it is a representation of causal dependencies graph between signals, given that it is learned correctly from a dataset. The equivalent classes of state space models which realize each DSF is the class of reference frames that share the same causal dependency graphs, and characterizing this equivalent class is essentially giving semantical meaning to each causal dependency graph.

\subsection{Semantics}

In computer science, we have a tradition of talking about formal semantics as interpretations of programs with correct syntax. In general, operational semantics, denotational semantics and axiomatic semantics are of our special interest because of their ubiquitous applications on program verification and validation. In particular, each of them means the following,[13]

- Operational semantics: The meaning of a construct is specified by the computation it induces when it is executed on a machine. In particular, how the effect of a computation is produced.

- Denotational semantics: Meanings are modeled by mathematical objects that represent the effect of executing the constructs. Thus only the effect is of interest, not how it is obtained.

- Axiomatic semantics: Specific properties of the effect of executing the constructs are expressed as assertions. Thus there may be aspects of the executions that are ignored.

The need of multiple semantics arise because of the need of verifying properties with different

level of details. Much like formal semantics of computer programs, LTI systems can be 
specified by various representations with different levels of computation details. Here is a list of semantics used for describing systems ordered from the most structurally informative to the least structurally informative, [5]

- Complete computational structure: An example is the state space representation, it describes the exact causal computation of how the states of a dynamical system would change with its current states.

- Partial Structure Representation: This kind of representations capture the abstract structure of a systems, through states partitioning or abstracting computations into causal relationships. The structural information captured in this kind of representations lie in between complete computational structure and manifest structure.

- Manifest structure: Much like the denotational semantics, it concerns only the behavior of a mathematical model instead of its sequence of computation. A well known example is the transfer function.

These three kinds of semantics are not exactly analogous to operational, denotational and axiomatic semantics in computer programs, but the sharing of similarities indicate the fact that there also exist different level of analysis for different semantic representation of dynamic systems. Specifically, a number of important analytical techniques have been developed for state space models, such as tests for controllability, observability and theory on minimal realization. Characterizing an equivalent class of state space models with the same DSF will allow us to examine these properties of some particular causal dynamic structures and discover new properties unique to signal structure of a system.

\subsection{Problem Decomposition and Reduction}

\subsubsection{Sufficient Conditions on State Transformations, $T$, that Preserve $(W, V)$}

Before diving into a discussion of sufficient conditions on a state transformation, $T$, that preserves $(W, V)$, we first define a new term structurally equivalent for convenience. 
Definition 2.3.1 (Structural Equivalence) Two systems are structurally equivalent if they are characterized by the same dynamical network function $(W, V)$.

In this subsection, we are interested in the sufficient conditions which would imply such equivalence, much like algebraic equivalence implies zero-state equivalence. A formal definition of algebraically equivalent can be found in [14],

Definition 2.3.2 Two continuous-time or discrete-time LTI systems

$$
\left\{\begin{array} { l } 
{ \dot { x } / x ^ { + } = A x + B u } \\
{ y = C x + D u }
\end{array} \text { or } \quad \left\{\begin{array}{l}
\dot{\bar{x}} / x^{+}=\bar{A} \bar{x}+\bar{B} u \\
y=\bar{C} \bar{x}+\bar{D} u
\end{array}\right.\right.
$$

respectively, are algebraically equivalent if there exists a nonsingular matrix $T$ such that

$$
\bar{A}:=T A T^{-1}, \quad \bar{B}:=T B, \quad \bar{C}:=C T^{-1}, \quad \bar{D}:=D .
$$

The corresponding map $\bar{x}=T x$ is called a similarity transformation or an equivalent transformation. Such transformation preserves the transfer function, which guarantees the same input-output behaviors of the black box. However, computations may be different inside the black box.

Intuitively, a sufficient condition on $T$ for preserving $(W, V)$ should be similar to but more restrictive than algebraic equivalence, since such a condition would partition a class of algebraically equivalent state space representations into subclasses of structurally equivalent SSRs characterized by the same $(W, V)$.

Theorem 2.3.1 (Sufficient Conditions for Preserving DNF) Restricting $T$ to be the following

$$
T=\left[\begin{array}{cc}
I & 0 \\
0 & T_{4}
\end{array}\right]
$$


is a sufficient condition of preserving dynamical network function of a state space representation on the state transformation $T$ [12].

Proof. The proof can be broken into two parts, first by showing that $\bar{W}=W$, then we will show that $\bar{V}=V$. Before start, let's remind ourselves of the definition of DNF

$$
\begin{gathered}
W(s)=A_{11}+A_{12}\left(s I-A_{22}\right)^{-1} A_{21}, \\
V(s)=A_{12}\left(s I-A_{22}\right)^{-1} B_{2}+B_{1} .
\end{gathered}
$$

Part 1: To show that $W(s)=\bar{W}(s)$, we can derive $\left(\bar{A}_{11}, \bar{A}_{12}, \bar{A}_{21}, \bar{A}_{22}\right)$ with the given $T$.

$$
\begin{aligned}
T A T^{-1} & =\left[\begin{array}{ll}
I & 0 \\
0 & T_{4}
\end{array}\right]\left[\begin{array}{ll}
A_{11} & A_{12} \\
A_{21} & A_{22}
\end{array}\right]\left[\begin{array}{cc}
I & 0 \\
0 & T_{4}^{-1}
\end{array}\right] \\
& =\left[\begin{array}{ll}
I & 0 \\
0 & T_{4}
\end{array}\right]\left[\begin{array}{ll}
A_{11} & A_{12} T_{4}^{-1} \\
A_{21} & A_{22} T_{4}^{-1}
\end{array}\right] \\
& =\left[\begin{array}{cc}
A_{11} & A_{12} T_{4}^{-1} \\
T_{4} A_{21} & T_{4} A_{22} T_{4}^{-1}
\end{array}\right] \\
& =\left[\begin{array}{ll}
\bar{A}_{11} & \bar{A}_{12} \\
\bar{A}_{21} & \bar{A}_{22}
\end{array}\right] .
\end{aligned}
$$

Therefore,

$$
\begin{aligned}
\bar{W}(s) & =\bar{A}_{11}+\bar{A}_{12}\left(s I-\bar{A}_{22}\right)^{-1} \bar{A}_{21} \\
& =A_{11}+A_{12} T_{4}^{-1}\left(s I-T_{4} A_{22} T_{4}^{-1}\right)^{-1} T_{4} A_{21} \\
& =A_{11}+A_{12} T_{4}^{-1}\left(T_{4}\left(s I-A_{22}\right) T_{4}^{-1}\right)^{-1} T_{4} A_{21} \\
& =A_{11}+A_{12}\left(s I-A_{22}\right)^{-1} A_{21} \\
& =W(s)
\end{aligned}
$$


Part 2: To show that $V(s)=\bar{V}(s)$, we derive $\left(\bar{B}_{1}, \bar{B}_{2}\right)$ with the given $T$.

$$
\begin{aligned}
T B & =\left[\begin{array}{ll}
I & 0 \\
0 & T_{4}
\end{array}\right]\left[\begin{array}{l}
B_{1} \\
B_{2}
\end{array}\right] \\
& =\left[\begin{array}{c}
B_{1} \\
T_{4} B_{2}
\end{array}\right] .
\end{aligned}
$$

Therefore,

$$
\begin{aligned}
\bar{V}(s) & =\bar{A}_{12}\left(s I-\bar{A}_{22}\right)^{-1} \bar{B}_{2}+\bar{B}_{1} \\
& =A_{12} T_{4}^{-1}\left(s I-T_{4} A_{22} T_{4}^{-1}\right)^{-1} T_{4} B_{2}+B_{1} \\
& =A_{12} T_{4}^{-1}\left(T_{4}\left(s I-A_{22}\right) T_{4}^{-1}\right)^{-1} T_{4} B_{2}+B_{1} \\
& =A_{12}\left(s I-A_{22}\right)^{-1} B_{2}+B_{1} \\
& =V(s) .
\end{aligned}
$$

The sufficient condition side of the proof is quite trivial, and therefore the major technical contribution of this work is to provide the necessary condition of the conjecture. We will briefly introduce the formal definition of dynamical structure function to justify the use of $\left(A, B,\left[\begin{array}{ll}I & 0\end{array}\right], 0\right)$ instead of $(A, B, C, D)$ in the proof. 


\subsubsection{Extended Definition of Dynamical Structure Function and Reduction to} $\left(A, B,\left[\begin{array}{ll}I & 0\end{array}\right], 0\right)$

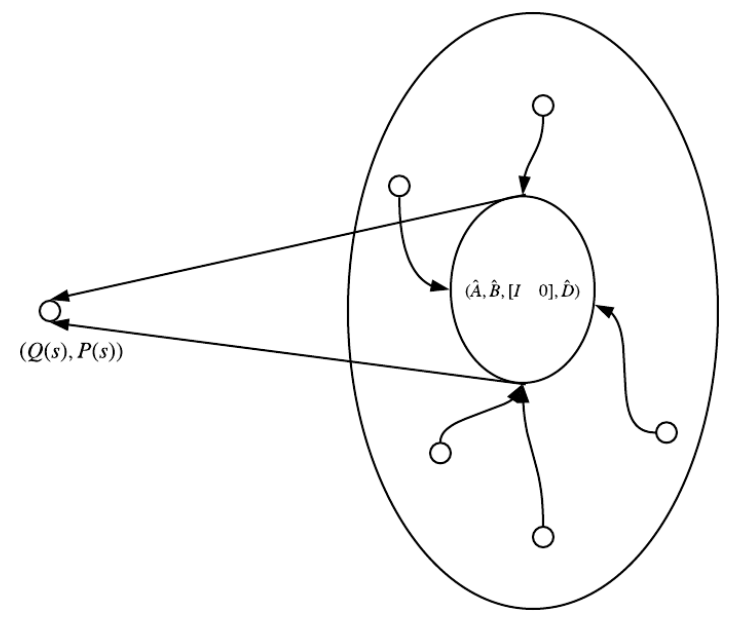

Figure 2.2: Graphical illustration of reducing $(A, B, C, D)$ to $\left(\hat{A}, \hat{B},\left[\begin{array}{ll}I & 0\end{array}\right], \hat{D}\right)$ when defining $(Q, P)$.

It is reasonable to ask at this point why we are justified to reduce the proof of structural preservation of similarity transforms on $(A, B, C, D)$ to $\left(A, B,\left[\begin{array}{ll}I & 0\end{array}\right], 0\right)$. [5] formalized a well defined notion of dynamical structure function and provides a 7 step procedure for deriving the signal structure. In other words, it defines the extended dynamical structure function, of an extended state space representation:

$$
\begin{aligned}
& \dot{x}=A x+B u+F \psi \\
& y=C x+D u+H \psi,
\end{aligned}
$$

where state variables $x \in \mathbb{R}^{n}$, output $y \in \mathbb{R}^{l}$, input $u \in \mathbb{R}^{m}$, with noise $\psi \in \mathbb{R}^{r}$. It turns out that the notion of problem reduction has been ingrained in the definition. To illustrate this, 
we preform the second step here with a regular state space model $(A, B, C, D)$ :

$$
\begin{aligned}
& \dot{x}=A x+B u \\
& y=C x+D u,
\end{aligned}
$$

where $C$ is a fat matrix with full row rank to indicate some possible loss of information from state space to output. Let a state transformation

$$
T=\left[\begin{array}{c}
C \\
E^{T}
\end{array}\right],
$$

where $E$ is a unitary matrix of any basis of $k e r(C)$. And now, $T^{-1}$ can be derived as:

$$
T^{-1}=\left[\begin{array}{ll}
C^{T}\left(C C^{T}\right)^{-1} & E
\end{array}\right]
$$

We can apply a change of basis on the system by $T$, let $z=T x$, where

$$
\hat{C}=C T^{-1}=\left[C C^{T}\left(C C^{T}\right)^{-1} \quad C E\right]=\left[\begin{array}{ll}
I & 0
\end{array}\right],
$$

and the new system is defined as:

$$
\begin{aligned}
{\left[\begin{array}{c}
\dot{z}_{1} \\
\dot{z_{2}}
\end{array}\right] } & =\left[\begin{array}{cc}
\hat{A}_{11} & \hat{A}_{12} \\
\hat{A}_{21} & \hat{A}_{22}
\end{array}\right]\left[\begin{array}{l}
z_{1} \\
z_{2}
\end{array}\right]+\left[\begin{array}{c}
\hat{B}_{1} \\
\hat{B}_{2}
\end{array}\right] u \\
y & =\left[\begin{array}{ll}
I & 0
\end{array}\right]\left[\begin{array}{l}
z_{1} \\
z_{2}
\end{array}\right]+\hat{D} u .
\end{aligned}
$$

Now, it is clear that there always exists a state transformation such that an arbitrary state space representation $(A, B, C, D)$ is algebraically equivalent to $\left(\hat{A}, \hat{B},\left[\begin{array}{ll}I & 0\end{array}\right], \hat{D}\right)$. Furthermore, it is upon this transformed state space representation the definition of dynamical structure 
function was built upon in [5]. Specifically, Dynamical network Function $(W, V)$ can be derived as:

$$
\begin{gathered}
W(s):=\hat{A}_{12}\left(s I-\hat{A}_{22}\right)^{-1} \hat{A}_{21}+\hat{A}_{11}, \\
V(s):=\hat{A}_{12}\left(s I-\hat{A}_{22}\right)^{-1} \hat{B}_{2}+\hat{B}_{1}
\end{gathered}
$$

just like what we had previously, and the dynamical structure function $(Q, P)$ is then defined as:

$$
\begin{aligned}
& Q(s):=(s I-\operatorname{diag}(W(s)))^{-1}(W(s)-\operatorname{diag}(W(s))), \\
& P(s):=(s I-\operatorname{diag}(W(s)))^{-1} V(s)+(I-Q(s)) \hat{D} .
\end{aligned}
$$

Again in [5], it has also been shown that signal structures of state space models under such definition is invariant to a set of block diagonal transformation $\left\{M \mid M=\left[\begin{array}{cc}I_{p \times p} & 0 \\ 0 & M_{22}\end{array}\right]\right\}$ and state permutation, which confirm the well-definedness of such definition. Since the $(W, V)$ is not affected by $D$, we shall let it be zero and focus our proof on the set of systems $\left(\bar{A}, \bar{B},\left[\begin{array}{ll}I & 0\end{array}\right], 0\right)$ and come back to it later.

\subsubsection{Similarity Transform of the Set of Reduced State Space Models}

Considering only the set of systems with $C=\left[\begin{array}{ll}I & 0\end{array}\right]$ and $D=0$, a state transformation $T$ between two similar systems in the set is forced to be of a certain form, that is:

$$
T=\left[\begin{array}{cc}
I & 0 \\
T_{3} & T_{4}
\end{array}\right]
$$


Lemma 2.3.2 Suppose two systems, $\left(A, B,\left[\begin{array}{ll}I & 0\end{array}\right], 0\right)$ with state variables $x$ and $\left(\bar{A}, \bar{B},\left[\begin{array}{ll}I & 0\end{array}\right], 0\right)$ with state variable $z$, are algebraically equivalent, then there exists an invertible state transformation $T=\left[\begin{array}{cc}I & 0 \\ T_{3} & T_{4}\end{array}\right]$ such that $z=T x$.

Proof. Suppose $C=\bar{C}=\left[\begin{array}{ll}I & 0\end{array}\right]$, and let $T=\left[\begin{array}{cc}T_{1} & T_{2} \\ T_{3} & T_{4}\end{array}\right]$. Then by evaluating $\bar{C}$,

$$
\begin{aligned}
& \bar{C}=C\left[\begin{array}{ll}
T_{1} & T_{2} \\
T_{3} & T_{4}
\end{array}\right] \\
& =\left[\begin{array}{ll}
I & 0
\end{array}\right]\left[\begin{array}{ll}
T_{1} & T_{2} \\
T_{3} & T_{4}
\end{array}\right] \\
& =\left[\begin{array}{ll}
T_{1} & T_{2}
\end{array}\right] \\
& =\left[\begin{array}{ll}
I & 0
\end{array}\right] \quad \text { (since } \bar{C}=\left[\begin{array}{ll}
I & 0
\end{array}\right] \text { is in the re- } \\
& \text { stricted subset of state space rep- } \\
& \text { resentations). }
\end{aligned}
$$




\section{Chapter 3}

\section{Main Result}

\subsection{Structural Parameters of a System}

We borrow the notion of Markov parameters [14] and extend it to an analogous notion called structural parameters for dynamical structure function in this subsection. This is going to

help prove the main theorem in a later section. The following is true from the definition of Laplace transform and matrix exponential:

$$
(s I-A)^{-1}=\mathcal{L}\left[e^{A t}\right]=: \mathcal{L}\left[\sum_{i=0}^{\infty} \frac{t^{i}}{i !} A^{i}\right]
$$

and

$$
(s I-A)^{-1}=\sum_{i=0}^{\infty} s^{-(i+1)} A^{i}
$$

through replacing $\mathcal{L}\left[\frac{t^{i}}{i !}\right]$ by $s^{-(i+1)}$. Hence, $W$ and $V$ can be rewritten in the following form,

$$
\begin{gathered}
W=A_{11}+\sum_{i=0}^{\infty} s^{-(i+1)} A_{12} A_{22}^{i} A_{21} \\
V=B_{1}+\sum_{i=0}^{\infty} s^{-(i+1)} A_{12} A_{22}^{i} B_{2} .
\end{gathered}
$$

Furthermore, the $i^{\text {th }}$ derivative of the impulse response of $W$ and $V$ when $t \rightarrow 0$ can be written as $A_{12} A_{22}^{i} A_{21}$ and $A_{12} A_{22}^{i} B_{2} \forall i \geq 1$ respectively. 
Definition 3.1.1 (Structural Parameters) The structural parameters of a state space representation of a system are

$$
\begin{array}{ll}
A_{11}, & A_{12} A_{22}^{i} A_{21}, \\
B_{1}, \quad A_{12} A_{22}^{i} B_{2}, \quad \forall i \geq 0 .
\end{array}
$$

Lemma 3.1.1 (Structural Parameters Preserve DNF) Two state space representations

$$
\left\{\begin{array} { l } 
{ \dot { x } = A x + B u } \\
{ y = C x + D u }
\end{array} \text { and } \quad \left\{\begin{array}{l}
\dot{\bar{x}}=\bar{A} \bar{x}+\bar{B} u \\
y=\bar{C} \bar{x}+\bar{D} u
\end{array}\right.\right.
$$

realize the same dynamical network function $(W, V)$ if and only if they have the same structural parameters, that is:

$$
\begin{array}{ll}
A_{11}=\bar{A}_{11}, & A_{12} A_{22}^{i} A_{21}=\bar{A}_{12} \bar{A}_{22}^{i} \bar{A}_{21}, \\
B_{1}=\bar{B}_{1}, & A_{12} A_{22}^{i} B_{2}=\bar{A}_{12} \bar{A}_{22}^{i} \bar{B}_{2},
\end{array}
$$

$\forall i \in \mathbb{N} \bigcup\{0\}$.

Proof. From equation (3.1) and (3.2), we know that two systems would have the same dynamical network function if they have the same structural parameters.

On the other hand, if two systems have the same dynamical network function, that is $W=\bar{W}$ and $V=\bar{V}$, then they must have the same $A_{11}$ and $B_{1}$. This can be shown by setting $s$ to infinity. Furthermore, since $W$ and $V$ are matrices of transfer functions, and equivalence of transfer functions implies equivalence of their impulse responses, we can conclude that the two systems have the same $A_{12} A_{22}^{i} A_{21}$ and $A_{12} A_{22}^{i} B_{2} \forall i$.

Furthermore, we can show that all strictly causal systems with zero transfer function $\hat{G}(s)=0$ if and only if they have zero Markov parameters. 


\section{Lemma 3.1.2 (Markov Parameters of Zero Systems)}

$$
\hat{G}(s)=C(s I-A)^{-1} B+D=0
$$

if and only if $C A^{i} B=D=0 \quad \forall i \geq 0$.

Proof. It is a special case of theorem 17.2 in [14] which states that Markov parameters of two systems are equal if and only if they are zero-state equivalent. Consider a zero system $\bar{A}, \bar{B}, \bar{C}, \bar{D}$ with transfer function $\hat{\bar{G}}(s)=0$, and Markov parameters $\bar{C} \bar{A}^{i} \bar{B}=\bar{D}=$ $0 \forall i \geq 0$, an example of such a system is $\bar{A}=\bar{B}=\bar{C}=\bar{D}=0$. Since the two systems $\hat{G}(s)=C(s I-A)^{-1} B+D$ and $\hat{\bar{G}}(s)=\bar{C}(s I-\bar{A})^{-1} \bar{B}+\bar{D}$ are zero-state equivalent, then by theorem 17.2 in [14], two realizations will also have the same Markov parameters, that is $C A^{i} B=\bar{C} \bar{A}^{i} \bar{B}=D=\bar{D}=0 \quad \forall i \geq 0$, and vise versa by the same theorem. Hence, the lemma is proven true.

\subsection{The Necessary and Sufficient Condition on Transformation $T$ that Preserves $(W, V)$}

Theorem 3.2.1 Two restricted LTI systems

$$
\left\{\begin{array} { l } 
{ \dot { x } = A x + B u } \\
{ y = [ \begin{array} { l l } 
{ I } & { 0 }
\end{array} ] x }
\end{array} \text { and } \quad \left\{\begin{array}{l}
\dot{\bar{x}}=\bar{A} \bar{x}+\bar{B} u \\
y=\left[\begin{array}{ll}
I & 0
\end{array}\right] \bar{x}
\end{array}\right.\right.
$$

respectively, are structurally equivalent if and only if there exists a nonsingular matrix $T=\left[\begin{array}{cc}I & 0 \\ T_{3} & T_{4}\end{array}\right]$ such that:

$$
A_{12} A_{22}^{i} T_{4}^{-1} T_{3}=0 \quad \forall i \geq 0 .
$$

Proof. From the previous section, we have shown that two systems are structurally equivalent if and only if they carry the same structural parameters. We are going to show that the two systems carry the same structural parameters if and only if there exists an invertible 


$$
T=\left[\begin{array}{cc}
I & 0 \\
T_{3} & T_{4}
\end{array}\right] \text { such that: }
$$

$$
A_{12}\left(s I-A_{22}\right)^{-1} T_{4}^{-1} T_{3}=0
$$

Let's define a $\bar{A}$ and $\bar{B}$ with a non-singular transformation $T$ such that:

$$
\begin{aligned}
\bar{A} & =T A T^{-1} \\
& =\left[\begin{array}{ll}
I & 0 \\
T_{3} & T_{4}
\end{array}\right]\left[\begin{array}{ll}
A_{11} & A_{12} \\
A_{21} & A_{22}
\end{array}\right]\left[\begin{array}{cc}
I & 0 \\
-T_{4}^{-1} T_{3} & T_{4}^{-1}
\end{array}\right] \\
& =\left[\begin{array}{cc}
I & 0 \\
T_{3} & T_{4}
\end{array}\right]\left[\begin{array}{ll}
A_{11}-A_{12} T_{4}^{-1} T_{3} & A_{12} T_{4}^{-1} \\
A_{21}-A_{22} T_{4}^{-1} T_{3} & A_{22} T_{4}^{-1}
\end{array}\right] \\
& =\left[\begin{array}{cc} 
& A_{11}-A_{12} T_{4}^{-1} T_{3} \\
-\left(T_{3} A_{12}+T_{4} A_{22}\right) T_{4}^{-1} T_{3} & \left(T_{3} A_{12}+T_{4} A_{22}\right) T_{4}^{-1} \\
\bar{A}_{11} & \bar{A}_{12} \\
\bar{A}_{21} & \bar{A}_{22}
\end{array}\right] .
\end{aligned}
$$


Also:

$$
\begin{aligned}
\bar{B} & =T B \\
& =\left[\begin{array}{ll}
I & 0 \\
T_{3} & T_{4}
\end{array}\right]\left[\begin{array}{l}
B_{1} \\
B_{2}
\end{array}\right] \\
& =\left[\begin{array}{c}
B_{1} \\
T_{3} B_{1}+T_{4} B_{2}
\end{array}\right] \\
& =\left[\begin{array}{c}
\bar{B}_{1} \\
\overline{B_{2}}
\end{array}\right]
\end{aligned}
$$

Part 1: $(\Rightarrow)$. Suppose the two systems carry the same structural parameters, that is:

$$
\begin{array}{ll}
A_{11}=\bar{A}_{11}, & A_{12} A_{22}^{i} A_{21}=\bar{A}_{12} \bar{A}_{22}^{i} \bar{A}_{21}, \\
B_{1}=\bar{B}_{1}, & A_{12} A_{22}^{i} B_{2}=\bar{A}_{12} \bar{A}_{22}^{i} \bar{B}_{2} .
\end{array}
$$

Then

$$
\begin{aligned}
A_{11} & =\bar{A}_{11} \\
A_{11} & =A_{11}-A_{12} T_{4}^{-1} T_{3} \\
A_{12} T_{4}^{-1} T_{3} & =0 .
\end{aligned}
$$

Therefore,

$$
A_{12} A_{22}^{i} T_{4}^{-1} T_{3}=0
$$

when $i=0$. 
Assume $A_{12} A_{22}^{i} T_{4}^{-1} T_{3}=0$ for all $0 \leq i \leq k$ for some $k \geq 0$. Then,

$$
\begin{aligned}
A_{12} A_{22}^{k} A_{21}= & \bar{A}_{12} \bar{A}_{22}^{k} \bar{A}_{21} \\
= & \left(A_{12} T_{4}^{-1}\right)\left(\left(T_{3} A_{12}+T_{4} A_{22}\right) T_{4}^{-1}\right)^{k}\left(T_{3} A_{11}+T_{4} A_{21}\right. \\
& \left.\quad-\left(T_{3} A_{12}+T_{4} A_{22}\right) T_{4}^{-1} T_{3}\right) \\
= & A_{12} A_{22}^{k} T_{4}^{-1}\left(T_{3} A_{11}+T_{4} A_{21}-\left(T_{3} A_{12}+T_{4} A_{22}\right) T_{4}^{-1} T_{3}\right) \\
= & A_{12} A_{22}^{k} T_{4}^{-1}\left(T_{4} A_{21}-T_{4} A_{22} T_{4}^{-1} T_{3}\right) \\
A_{12} A_{22}^{k+1} T_{4}^{-1} T_{3}= & 0 .
\end{aligned}
$$

Notice that $\left(A_{12} T_{4}^{-1}\right)\left(\left(T_{3} A_{12}+T_{4} A_{22}\right) T_{4}^{-1}\right)^{k}$ is evaluated to $A_{12} A_{22}^{k} T_{4}^{-1}$ because any terms in the expansion of $\left(A_{12} T_{4}^{-1}\right)\left(\left(T_{3} A_{12}+T_{4} A_{22}\right) T_{4}^{-1}\right)^{k}$ composed with at least one $T_{3} A_{12} T_{4}^{-1}$ will have the form $A_{12} A_{22}^{l} T_{4}^{-1} T_{3} A_{12} \Phi$ where $0 \leq l<k$, and are evaluated to 0 by the inductive assumption.

Therefore, by principle of mathematical induction,

$$
A_{12} A_{22}^{i} T_{4}^{-1} T_{3}=0 \quad \forall i \geq 0
$$

if the two systems carry the same structural parameters.

Part 2: $(\Leftarrow)$. By definition of $\bar{B}$, we have $B_{1}=\bar{B}_{1}$.

Suppose:

$$
A_{12} A_{22}^{i} T_{4}^{-1} T_{3}=0 \quad \forall i \geq 0
$$


then by definition of $\bar{A}_{11}, \bar{A}_{11}=A_{11}-A_{12} T_{4}^{-1} T_{3}=A_{11}$, also:

$$
\begin{aligned}
\bar{A}_{12} \bar{A}_{22}^{i} \bar{A}_{21}= & \left(A_{12} T_{4}^{-1}\right)\left(\left(T_{3} A_{12}+T_{4} A_{22}\right) T_{4}^{-1}\right)^{i}\left(T_{3} A_{11}+T_{4} A_{21}\right. \\
& \left.-\left(T_{3} A_{12}+T_{4} A_{22}\right) T_{4}^{-1} T_{3}\right) \\
= & A_{12} A_{22}^{i} T_{4}^{-1}\left(T_{3} A_{11}+T_{4} A_{21}-\left(T_{3} A_{12}+T_{4} A_{22}\right) T_{4}^{-1} T_{3}\right) \\
= & A_{12} A_{22}^{i} T_{4}^{-1}\left(T_{4} A_{21}-T_{4} A_{22} T_{4}^{-1} T_{3}\right) \\
= & A_{12} A_{22}^{i} A_{21} .
\end{aligned}
$$

The same argument applies to prove $\bar{A}_{12} \bar{A}_{22}^{i} \bar{B}_{2}=A_{12} A_{22}^{i} B_{2}$ by replacing $A_{21}$ with $B_{2}$ and $\bar{A}_{21}$ with $\bar{B}_{2}$.

Therefore, the two systems carry the same structural parameters, that is:

$$
\begin{array}{ll}
A_{11}=\bar{A}_{11}, & A_{12} A_{22}^{i} A_{21}=\bar{A}_{12} \bar{A}_{22}^{i} \bar{A}_{21}, \\
B_{1}=\bar{B}_{1}, & A_{12} A_{22}^{i} B_{2}=\bar{A}_{12} \bar{A}_{22}^{i} \bar{B}_{2} .
\end{array}
$$

With lemma (3.1.1), we have shown that (structural equivalence) $\Leftrightarrow$ (same structural parameters $) \Leftrightarrow\left(A_{12} A_{22}^{i} T_{4}^{-1} T_{3}=0\right)$. Therefore, two systems are structurally equivalent if and only if

$$
A_{12} A_{22}^{i} T_{4}^{-1} T_{3}=0 \quad \forall i \geq 0
$$

It turns out that this condition has to do with the orthogonality between hidden dynamics of the system, that is $A_{12}$ and $A_{22}$, and the reduced components of the state transformation, $T_{4}^{-1}$ and $T_{3}$.

We can further simplify the condition by applying lemma (3.1.2). We know (3.3) is true if and only if

$$
A_{12}\left(s I-A_{22}\right)^{-1} T_{4}^{-1} T_{3}=0
$$

is true by substituting $A_{12}, A_{22}, T_{4}^{-1} T_{3}$ into $C, A, B$. 


\subsection{Equivalent classes of $(W, V)$ characterized by the same $(Q, P)$}

In this section, we will cover the condition for constructing equivalent classes of dynamical network functions which are characterized by the same dynamical structure function $(Q, P)$.

Theorem 3.3.1 Two dynamical network functions $(W, V)$ and $(\bar{W}, \bar{V})$ realize the same dynamical structure function $(Q, P)$ if and only if there exist a transformation $L$ such that

$$
L V=\bar{V}, \quad L(W-\operatorname{diag}(W))=\bar{W}-\operatorname{diag}(\bar{W}) .
$$

Furthermore, $L=(s I-\operatorname{diag}(\bar{W}(s)))(s I-\operatorname{diag}(W(s)))^{-1}$.

Proof. By definition $(2.2),(Q, P)$ are defined as

$$
\begin{aligned}
& Q(s)=(s I-\operatorname{diag}(W(s)))^{-1}(W(s)-\operatorname{diag}(W(s))), \\
& P(s)=(s I-\operatorname{diag}(W(s)))^{-1} V(s)+(I-Q(s)) \hat{D} .
\end{aligned}
$$

Let $D_{s W}=(s I-\operatorname{diag}(W(s)))$ and $\bar{D}_{s W}=(s I-\operatorname{diag}(\bar{W}(s))) .(W, V)$ and $(\bar{W}, \bar{V})$ realize the same dynamical structure function $(Q, P)$ if and only if they have the same $\hat{D}$ and derive the same $(Q, P)$, that is:

$$
\begin{aligned}
D_{s W}^{-1}(W(s)-\operatorname{diag}(W(s))) & =\bar{D}_{s W}^{-1}(\bar{W}(s)-\operatorname{diag}(\bar{W}(s))) \\
D_{s W}^{-1} V(s) & =\bar{D}_{s W}^{-1} \bar{V}(s) .
\end{aligned}
$$

Therefore,

$$
\begin{aligned}
(\bar{W}(s)-\operatorname{diag}(\bar{W}(s))) & =\bar{D}_{s W} D_{s W}^{-1}(W(s)-\operatorname{diag}(W(s))) \\
\bar{V}(s) & =\bar{D}_{s W} D_{s W}^{-1} V(s) .
\end{aligned}
$$


Set $L:=\bar{D}_{s W} D_{s W}^{-1}$, and it's clear that it is equivalent to the existence of a transformation $L$ such that

$$
\begin{aligned}
(\bar{W}(s)-\operatorname{diag}(\bar{W}(s))) & =L(W(s)-\operatorname{diag}(W(s))) \\
\bar{V}(s) & =L V(s) .
\end{aligned}
$$

Notice that $\hat{D}$ is not specified by $(W, V)$, therefore, determination of equivalent $(Q, P)$ realization from $(W, V)$ does not depend on the value of $\hat{D}$. 


\section{Chapter 4}

\section{Conclusion}

In this work, we have found a necessary and sufficient condition on a state transformation $T$ such that the dynamical network function $(W, V)$ might preserve. And it turns out that this condition has to do with the transfer function characterized by the strictly causal system $\left(A_{22}, T_{4}^{-1} T_{3}, A_{12}, 0\right)$. This finding will allow us to answer questions related to optimal stabilizing distributed controllers design and future theories on state space model, dynamical structure function and transfer function.

Furthermore, we could develop metrics to characterize how far a transformation might bring a state space model away from its original causal structure. The $\mathcal{H}_{2}$ norm of the

transfer function $A_{12}\left(s I-A_{22}\right)^{-1} T_{4}^{-1} T_{3}$ would be an example. Such metric for measuring structural difference between models in terms of similarity transform is different from other conventional methods in graph theory, such as comparing graphs Laplacian matrices. This method accounts for the behavior of systems under abstraction, a.k.a. removal of nodes, which might provide a new perspectives on graph related future research. 


\section{References}

[1] M. Araki and M. Saeki. A quantitative condition for the well-posedness of interconnected dynamical systems. IEEE Transactions on Automatic Control, 28(5):569-577, May 1983. ISSN 0018-9286. doi: 10.1109/TAC.1983.1103282.

[2] B. Bamieh and L. Giarre. Identification of linear parameter varying models. In 38th IEEE Conference on Decision and Control (CDC), volume 2, pages 1505-1510, Dec 1999. doi: 10.1109/CDC.1999.830205.

[3] Tian Qi Chen, Yulia Rubanova, Jesse Bettencourt, and David K. Duvenaud. Neural ordinary differential equations. Computing Research Repository (CoRR), abs/1806.07366, 2018. URL http://arxiv.org/abs/1806.07366.

[4] V. Chetty. Necessary and sufficient informativity conditions for robust network reconstruction using dynamical structure functions. M.S. Thesis, Brigham Young Univ., Provo, UT, 2012.

[5] V. Chetty and S. Warnick. Network semantics of dynamical systems. In 54th IEEE Conference on Decision and Control (CDC), pages 1557-1562, Dec 2015. doi: 10.1109/ CDC.2015.7402432.

[6] V. Chetty, J. Eliason, and S. Warnick. Passive reconstruction of non-target-specific discrete-time LTI systems. In American Control Conference (ACC), pages 66-71, Jul 2016. doi: 10.1109/ACC.2016.7524893.

[7] V. Chetty, N. Woodbury, J. Brewer, K. Lee, and S. Warnick. Applying a passive network reconstruction technique to twitter data in order to identify trend setters. In 2017 IEEE Conference on Control Technology and Applications (CCTA), pages 1344-1349, Aug 2017. doi: 10.1109/CCTA.2017.8062645.

[8] Vasu Chetty and Sean Warnick. Meanings and applications of structure in networks of dynamic systems. Computing Research Repository (CoRR), abs/1406.1844, 2014. URL http://arxiv.org/abs/1406.1844. 
[9] A. Dankers, P. M. J. Van den Hof, X. Bombois, and P. S. C. Heuberger. Identification of dynamic models in complex networks with prediction error methods: Predictor input selection. IEEE Transactions on Automatic Control, 61(4):937-952, Apr 2016. ISSN 0018-9286. doi: 10.1109/TAC.2015.2450895.

[10] Emilio Frazzoli and Munther Dahleh. Dynamics systems and control 6.241j. Massachusetts Institute of Technology: MIT OpenCourseWare https://ocw.mit.edu, Spring 2011. License: Creative Commons BY-NC-SA.

[11] J. Goncalves and S. Warnick. Necessary and sufficient conditions for dynamical structure reconstruction of LTI networks. IEEE Transactions on Automatic Control, 53(7):16701674, Aug 2008. ISSN 0018-9286. doi: 10.1109/TAC.2008.928114.

[12] J. Goncalves, R. Howes, and S. Warnick. Dynamical structure functions for the reverse engineering of LTI networks. In 46th IEEE Conference on Decision and Control (CDC), pages 1516-1522, Dec 2007. doi: 10.1109/CDC.2007.4434406.

[13] Flemming Nielson Hanne Riis Nielson. Semantics with applications a formal introduction, chapter 1. John Wiley \& Sons, July 1999.

[14] João Pedro Hespanha. Linear Systems Theory, pages 37, 40, 158-161. Princeton university press, 2009.

[15] R. E. Kalman. Mathematical description of linear dynamical systems. Journal of the Society for Industrial and Applied Mathematics Series A Control, 1(2):152-192, 1963. doi: 10.1137/0301010. URL https://doi.org/10.1137/0301010.

[16] Helmut Ltkepohl. New Introduction to Multiple Time Series Analysis, chapter 3.6. Springer Publishing Company, Incorporated, 2007. ISBN 3540262393, 9783540262398.

[17] D. Materassi and G. Innocenti. Topological identification in networks of dynamical systems. IEEE Transactions on Automatic Control, 55(8):1860-1871, Aug 2010. ISSN 0018-9286. doi: 10.1109/TAC.2010.2042347.

[18] D. Materassi and G. Innocenti. Topological identification in networks of dynamical systems. IEEE Transactions on Automatic Control, 55(8):1860-1871, Aug 2010. ISSN 0018-9286. doi: 10.1109/TAC.2010.2042347.

[19] Tomas Mikolov, Martin Karafiát, Lukás Burget, Jan Cernocký, and Sanjeev Khudanpur. Recurrent neural network based language model. Proceedings of the 11th Annual 
Conference of the International Speech Communication Association, INTERSPEECH 2010, 2:1045-1048, Jan 2010.

[20] P. E. Par and S. Warnick. Information cost for the state reconstruction of linear time invariant systems. In 54th IEEE Conference on Decision and Control (CDC), pages 875-880, Dec 2015. doi: 10.1109/CDC.2015.7402339.

[21] Judea Pearl. Causality: Models, Reasoning and Inference, pages 30-38, 331-358. Cambridge University Press, New York, NY, USA, 2nd edition, 2009. ISBN 052189560X, 9780521895606 .

[22] C. J. Quinn, N. Kiyavash, and T. P. Coleman. Directed information graphs. IEEE Transactions on Information Theory, 61(12):6887-6909, Dec 2015. ISSN 0018-9448. doi: 10.1109/TIT.2015.2478440.

[23] A. Rai and S. Warnick. A technique for designing stabilizing distributed controllers with arbitrary signal structure constraints. In European Control Conference (ECC), pages 3282-3287, Jul 2013. doi: 10.23919/ECC.2013.6669177.

[24] J. C. Willems. The behavioral approach to open and interconnected systems. IEEE Control Systems, 27(6):46-99, Dec 2007. ISSN 1066-033X. doi: 10.1109/MCS.2007. 906923.

[25] N. Woodbury. Network reconstruction and vulnerability analysis of financial networks. M.S. Thesis, Brigham Young Univ., Provo, UT, 2017.

[26] E. Yeung, J. Gonalves, H. Sandberg, and S. Warnick. Representing structure in linear interconnected dynamical systems. In 49th IEEE Conference on Decision and Control (CDC), pages 6010-6015, Dec 2010. doi: 10.1109/CDC.2010.5718109. 